\title{
Importance of vaccine history in suspected measles.
}

\section{Case Report}

A previously healthy, 19-month-old girl presented to a tertiary paediatric hospital emergency department with a two day history of generalised non-pruritic maculopapular rash, initially noted on her face and shoulders. She was otherwise well with no recent measured fever (though was reported to have "felt hot" at home), nor was there cough or coryza. She was seen by her general practitioner (GP) the day prior with a similar presentation, with a diagnosis made of a viral exanthema. Her parents were concerned the rash was spreading. Emergency department staff initially understood the child and her mother had recently returned from a trip to Sri Lanka. The child was attending day-care five days per week, but parents were unaware of any other child unwell with a rash, nor any other unwell contacts.

On examination, the child was alert and afebrile, reported to be slightly unsettled. Her pulse rate was 120 beats per minute, respiratory rate 30 breaths per minute and oxygen saturation $100 \%$ on room air. Her weight was $11 \mathrm{~kg}$ ( $50^{\text {th }}$ centile). She had an erythematous, blanching, morbilliform rash on her face, torso and back, which was consistent with measles exanthema. Her throat was mildly erythematous. Clinical examination was otherwise unremarkable.

Given the rash and travel history, measles was suspected, and the child was placed in isolation within a negative-pressure room with precautions appropriate for suspected measles, immediately after triage. A throat swab was collected and sent for reverse transcriptase-polymerase chain reaction (PCR) testing for measles and rubella RNA.

It was further noted in the record that this child's routine immunisations, according to the National Immunisation Program schedule, normally administered at 12 months (Haemophilus influenzae type B, Neisseria meningitidis serogroup C, measles, mumps and rubella immunisations) had been delayed, and she had in fact received these 12 days prior to the rash developing.

After a dose of ibuprofen, the child was more settled and judged well enough to be discharged home, with advice to follow up with her GP in two days for a physical examination and review of throat swab

This is the author manuscript accepted for publication and has undergone full peer review but has not been through the copyediting, typesetting, pagination and proofreading process, which may lead to differences between this version and the Version of Record. Please cite this article as doi: 10.1111/jpc.14647

This article is protected by copyright. All rights reserved. 
results. Given the clinical state of the child, history, lack of progression and setting, the Public Health Unit regarded home care at this stage as most appropriate.

Her throat swab results returned positive the following day for measles virus RNA.

The family was promptly contacted by the public health team, informed about the result and advised to remain in isolation at home from the public until the rash resolved. At this stage the hospital Infectious Diseases (ID) team was also contacted to advice on the case and consider whether any contact tracing within the hospital was required. The travel history was reviewed by the ID team and it was clarified that the child's mother had recently returned from a trip from Sri Lanka, but the child did not accompany her. The ID team then concluded this was likely vaccine-associated rash rather than measles infection. The throat swab sample was sent for further analysis to the World Health Organisation (WHO) Measles Reference Laboratory at the Victorian Infectious Diseases Reference Laboratory (VIDRL), which confirmed measles vaccine strain (genotype A). It was concluded that the rash was a vaccine-induced measles rash. The child recovered uneventfully.

\section{Key points}

1. Vaccine-induced rash occurs in 5\% of patients within 5-12 days of receiving a measlescontaining vaccine.

2. Testing for measles is not recommended if a child has been immunised with a measlescontaining vaccine in the preceding 8 weeks, in the absence of other risk factors.

3. Genotyping can be used to differentiate wild-type measles from vaccine-strain measles. Vaccine-strain measles does not cause infection and isolation precautions are not required.

4. The simultaneous detection of other vaccine viruses suggests that infection is unlikely.

\section{Discussion}

Measles infection, previously almost universal in childhood, is now uncommon in regions with high vaccination rates. Measles vaccinations were first registered in Australia in 1968 and the combined

This article is protected by copyright. All rights reserved. 
measles, mumps rubella vaccine (MMR) was added to the national funded immunisation schedule in $1989^{8}$. The WHO declared measles to be eliminated in Australia in $2014^{2}$. MMR vaccine is very effective for prevention of measles, providing immunity to the wild type strain of measles in up to $99 \%$ of recipients after the second dose $^{3}$. Despite this, outbreaks and measles cases continue occur sporadically, secondary to importation from endemic areas.

Measles virus is highly contagious and spreads through respiratory droplets, with viability for up to 2 hours in confined airspaces. It is characterized by fever and malaise, followed by cough, coryza and conjunctivitis, and a rash which typically begins on the face and spreads to become generalised, developing 3-5 days after these first symptoms ${ }^{1}$. Measles may be complicated by otitis media, pneumonia or diarrhoea. Subacute sclerosing panencephalitis (SSPE) is a rare but fatal late complication of measles, characterised by progressive neuronal degeneration and demyelination, typically occurring 7 to 10 years after the initial infection ${ }^{4}$.

Following vaccination, fever and or rash may occur in up to $5 \%$ of recipients, with onset typically 5- 12 days following administration of the vaccine ${ }^{4}$. The rash is sometimes referred to as vaccine- induced "measles" and is due to the measles vaccine virus, but does not cause infectious measles in others. However, isolation may still be required in these circumstances for children admitted to hospital, as fever and rash after administration of the MMR vaccine may also coincidentally occur with infection due to wild-type measles or infection with other viruses such as parvovirus, enterovirus or adenovirus $^{5}$. Rapid assays for distinguishing vaccine strain (genotype A) from wild type strains are now available ${ }^{9}$. Confirmation is always required using definitive assays available in WHO national reference laboratories ${ }^{9}$.

Isolation precautions play a crucial role in preventing exposure from genuine measles cases in a hospital setting. In a case of unrecognized measles exposure at a Sydney hospital in 2009, 111 individuals, including patients, family members and health-care workers (HCW) were potentially exposed. A significant amount of time and effort was required, involving physicians, infection control, public health and outpatient nursing staff as well as laboratory staff, to make phone calls, process serology specimens, administer normal human immunoglobulin (NHIG) or MMR vaccines and mail out letters ${ }^{7}$. In our case, however, had staff been aware of the significance of the vaccination history and

This article is protected by copyright. All rights reserved. 
correct travel history the efforts of many healthcare staff and consequent expense may have been avoided.

\section{Conclusions}

The occurrence of a rash 5-12 days following administration of measles-containing vaccine may indicate measles vaccine-induced rash. This condition is mild and self-limiting. Carers of vaccine recipients should be advised about these symptoms in the period 5-12 days following vaccination. Clinicians and vaccine-providers should also be aware of this. Testing for measles is generally not recommended if a child has been immunized with a measles-containing vaccine in the preceding eight

weeks. In rare cases where it is important to differentiate between wild-type and vaccine-strain measles in clinical samples, genotyping may be performed.

\section{Multiple choice questions}

1. When is a person with measles infection considered to be infectious?
a. 7-18 days after the onset of symptoms.
b. From the beginning of the prodromal period until 4 days after the onset of rash.
c. 1-3 days before development of rash.
d. 14 days before development of rash.
e. 7-10 days after the start of fever.

Answer: b. Measles has an average incubation period of 10 days (7-18 days) before the start of the fever and around 14 days prior to the onset of the rash. It is infectious from the beginning of the prodromal symptomatic period up to 4 days after the appearance of rash. Koplik spots in the pharynx, pathognomonic of measles, may appear 1-2 days before the rash

This article is protected by copyright. All rights reserved. 
2. Which of the following is often the first sign of measles observed by parents?
a. Koplik spots
b. High fever
c. Rash
d. Cough
e. Conjunctivitis

Answer: b. The first sign of measles in a child is usually high fever, followed by cough, coryza and conjunctivitis. This lasts 2-4 days, and is then followed by a rash. Koplik spots are seen on the buccal mucosa 1-2 days prior to the rash. Although pathognomonic, they are not often observed by parents or doctors.

3. What type of isolation/precautions are required for hospitals upon suspicion of measles?
a. Strict handwashing is generally sufficient to prevent transmission
b. No special or additional precautions are required while awaiting genotyping to differentiate vaccine- and wild-type strains
c. Gown and gloves are to be worn by healthcare staff
d. Airborne precautions, with isolation of the patient in a negative-pressure room if possible
e. Post-exposure prophylaxis with immunoglobulin for all non-immune staff

Answer: $d$. All suspected cases of measles should be isolated as soon as possible: Patients should be identified at the triage desk, be fitted with a mask, and placed immediately in a separate room, preferably with negative-pressure ventilation. Airborne precautions should be maintained until the child leaves hospital or is considered non-infectious (4 days after the onset of rash). Post-exposure prophylaxis with measles immunisation or immunoglobulin is only required for non-immune staff/contacts who are exposed, which should be avoidable in the hospital setting if these precautions are followed.

This article is protected by copyright. All rights reserved. 


\section{References}

1. Measles. The Australian Immunization Handbook $10^{\text {th }}$ Edition. Australian Government. Department of Health. Available online at:

http://www.immunise.health.gov.au/internet/immunise/publishing.nsf/Content/Handbook 10-home handbook10part4 handbook10-4-9\#4-9-1

2. Maclntyre CR et al. Measles control in Australia- threats, opportunity and future needs. Vaccine 2018; 36 (30): 4393-4398.

3. Strebel PM, Papania MJ, Dayan GH, Halsey NA. Measles vaccine. In: Plotkin SA, Orenstein WA, Offit PA, eds. Vaccines. 5th ed. Philadelphia, PA: Saunders Elsevier, 2008.

4. Centers for Disease Control and Prevention (CDC). Measles. In: Atkinson W, Wolfe C, Hamborsky J, eds. Epidemiology and prevention of vaccine-preventable diseases. 12th ed. Washington, D.C.: Public Health Foundation, 2011.

5. I Davidkin et al. Etiology of Measles- and Rubella-like Illnesses in Measles, Mumps, and Rubella-Vaccinated Children. The Journal of Infectious Diseases. 1998; 178 (6): 1567-1570.

6. CY Ting, Nancy WS Tee and KC Thoon. Could a fever and rash after the measles, mumps and rubella vaccination indicate wild-type measles? Acta Paediatrica. 2014; 104: 232-234.

7. Bowen $A C$, Ferson MJ and Palasanthiran P. Consequences of an unrecognized measles exposure in an emergency department. Emergency Medicine Australasia 2009; 21: 491-496.

8. National Centre for Immunisation Research and Surveillance. Significant events in measles, mumps and rubella vaccination practice in Australia. March 2016. Available online at: http://www.ncirs.edu.au/assets/provider_resources/history/Measles-mumps-rubellahistory-March-2016.pdf

9. K Thapa, JA Ellem, K Basile et al. Simultaneous co-detection of wild type and vaccine strain measles virus using the BD MAX system. Pathology. June 2018; 50 (4): 450-454.

This article is protected by copyright. All rights reserved. 


\section{University Library}

\section{- M M I E E R VA A gateway to Melbourne's research publications}

Minerva Access is the Institutional Repository of The University of Melbourne

Author/s:

Ewe, YH;Palasanthiran, P;Rawlinson, WD;McMullan, B

Title:

Importance of vaccine history in suspected measles

Date:

2020-05-01

Citation:

Ewe, Y. H., Palasanthiran, P., Rawlinson, W. D. \& McMullan, B. (2020). Importance of vaccine history in suspected measles. JOURNAL OF PAEDIATRICS AND CHILD HEALTH, 56 (5), pp.804-805. https://doi.org/10.1111/jpc.14647.

Persistent Link:

http://hdl.handle.net/11343/286500 